Bull. Austral. Math. Soc.

$46 \mathrm{~A} 08,03 \mathrm{E} 10$

VOL. 51 (1995) [521-528]

\title{
THE FIT AND FLAT COMPONENTS OF BARRELLED SPACES
}

\author{
Stephen A. Saxon and Ian Tweddle
}

\begin{abstract}
The Splitting Theorem says that any given Hamel basis for a (Hausdorff) barrelled space $E$ determines topologically complementary subspaces $E_{C}$ and $E_{D}$, and that $E_{C}$ is flat, that is, contains no proper dense subspace. By use of this device it was shown that if $E$ is non-flat it must contain a dense subspace of codimension at least $\aleph_{0}$; here we maximally increase the estimate to $\aleph_{1}$ under the assumption that the dominating cardinal $\mathfrak{d}$ equals $\aleph_{1}$ [strictly weaker than the Continuum Hypothesis (CH)]. A related assumption strictly weaker than the Generalised $\mathrm{CH}$ allows us to prove that $E_{D}$ is fit, that is, contains a dense subspace whose codimension in $E_{D}$ is $\operatorname{dim}\left(E_{D}\right)$, the largest imaginable. Thus the two components are extreme opposites, and $E$ itself is fit if and only if $\operatorname{dim}\left(E_{D}\right) \geqslant \operatorname{dim}\left(E_{C}\right)$, in which case there is a choice of basis for which $E_{D}=E$. Morover, $E$ is non-flat (if and) only if the codimension of $E^{\prime}$ is at least $2^{K_{1}}$ in $E^{*}$. These results ensure latitude in the search for certain subspaces of $E^{*}$ transverse to $E^{\prime}$, as in the barrelled countable enlargement (BCE) problem, and show that every non-flat $G M$-space has a BCE.
\end{abstract}

\section{INTRODUCTION}

The codensity character of a topological vector space $E$ (here always assumed to be Hausdorff) is the supremum of the set of codimensions of all dense subspaces in $E$. The dimension of $E$ is an obvious upper bound. When $E$ contains a dense subspace with codimension equal to the dimension of $E$ we say $E$ is fit. At the other extreme, when the only dense subspace of $E$ is $E$ itself we say $E$ is flat. Clearly $E$ is flat if and only if its (continuous) dual $E^{\prime}$ and its algebraic dual $E^{*}$ coincide, $E$ has its strongest locally convex topology if and only if $E$ is flat and barrelled, and the only space that is both fit and flat is the space consisting of the single element 0 . These and intermediate ideas and examples are found in [5].

In the present note we show that, under an axiomatic assumption strictly weaker than the Generalised Continuum Hypothesis (GCH), every barrelled space can be expressed as the topological direct sum of two subspaces, one of which is fit while the

Received 7 September 1994

The first author thanks Professors D. Cenzer and J. Larson for substantive discussions on the Settheoretic Lemma. This paper was prepared in part while the second author was visiting the University of Florida. He gratefully acknowledges support from the University of Florida and the Carnegie Trust for the Universities of Scotland.

Copyright Clearance Centre, Inc. Serial-fee code: 0004-9729/95 \$A2.00+0.00. 
other is flat. Consequently, the codensity character of a barrelled space $E$ is always attained as the maximum of its defining set (that is, $E$ is firm [5]) and is either 0 or at least $\aleph_{1}$. Under our axiomatic assumption this optimally improves the estimate of $N_{0}$ given in [3]. Moreover, the simple method we used in [5] is the only way of constructing barrelled spaces that are neither fit nor flat. We also apply the result to obtain important information about the codimension of the dual in the algebraic dual of a barrelled space and to extend the class of spaces for which it can be shown that a barrelled countable enlargement exists [10].

If $A$ is a subset of a topological vector space, $s p(A)$ denotes the linear span of $A$, with closure $\overline{s p}(A)$. For convenience we state the following theorem from [3], which is crucial for our work.

Splitting Theorem. Let $E$ be a barrelled space and let $\left\{x_{i}, f_{i}\right\}_{i \in B}$ be a biorthogonal system in $E \times E^{*}$ whose first coordinates form a Hamel basis in $E$. If

$$
E_{C}=s p\left(\left\{x_{i}: i \in B, f_{i} \in E^{\prime}\right\}\right) \text { and } E_{D}=s p\left(\left\{x_{i}: i \in B, f_{i} \notin E^{\prime}\right\}\right)
$$

then $E$ is the topological direct sum of $E_{C}$ and $E_{D}$, and $E_{C}$ has its strongest locally convex topology.

\section{AN AXIOMATIC ASSUMPTION}

If $A$ is any set we write $|A|$ for its cardinality, which we consider to be the set of all ordinals of smaller size. For an infinite cardinal $\mu$ we employed in [5] a Condition (1) weaker than the GCH, namely that $2^{<\mu}=\mu$. Here we shall make use of the following yet weaker axiomatic condition:

Condition (2). Given any set $A$ of cardinality less than $\mu$ there exists a set $\mathcal{F}$ of functions $f$ from $A$ into the set $\mathbb{N}$ of natural numbers such that the cardinality of $\mathcal{F}$ is at most $\mu$ and such that for any $g: A \rightarrow \mathbb{N}$ there exists $f \in \mathcal{F}$ such that $f(a)>g(a)$ for all $a \in A$.

REMARKS. (i) If $\aleph_{0} \leqslant|A|<\mu$ then under GCH the set of all functions $g: A \rightarrow \mathbb{N}$ has cardinality $\aleph_{0}^{|A|} \leqslant\left(2^{\aleph_{0}}\right)^{|A|}=2^{|A|} \leqslant \mu$; if $A$ is finite then this set of functions is countable. Thus GCH implies Condition (2) for all $\mu>\aleph_{0}$. (The case $\mu=\aleph_{0}$ does not require any assumptions.)

(ii) In the case $\mu=\aleph_{1}$, the set $A$ must be countable and so it can be regarded as $\mathbb{N}$ if it is infinite. Condition (2) may then be reformulated as

Condition ( $\left.2^{\prime}\right)$. There exists a set $\mathcal{F}$ of functions $f$ from $\mathbb{N}$ into $\mathbb{N}$ such that the cardinality of $\mathcal{F}$ is at most $\aleph_{1}$ and such that for any $g: \mathbb{N} \rightarrow \mathbb{N}$ there exists $f \in \mathcal{F}$ such that $f(n)>g(n)$ for all $n \in \mathbb{N}$. 
In fact, the minimum cardinality for such an $\mathcal{F}$ is the dominating cardinal $\mathfrak{d}$ ([1, Section 3]; see also $[7,9])$. It is easily seen that $\aleph_{1} \leqslant \mathfrak{d} \leqslant c$, and any combination of strict or non-strict inequalities is consistent with the usual ZFC set theory $[1$, Section 5]; Condition ( $\left.2^{\prime}\right)$ is equivalent to $\mathfrak{d}=\aleph_{1}$.

Set-theoret IC Lemma. Let $\mu$ be a fixed infinite cardinal. Condition (2) holds for $\mu$ if and only if for any fixed set $B$ of cardinality $\mu$ there exists a family $\left\{\left\{B_{\alpha 1}, B_{\alpha 2}, \ldots\right\}\right\}_{\alpha \in \mu}$ of partitions $\left\{B_{\alpha 1}, B_{\alpha 2}, \ldots\right\}$ of $B$ into sets $B_{\alpha p}$, each of cardinality $\mu$, such that, given any $g: \mu \rightarrow \mathbb{N}$ and any $\alpha_{0} \in \mu$,

$$
B \backslash \bigcup\left\{B_{\alpha k}: \alpha \leqslant \alpha_{0}, k \leqslant g(\alpha)\right\} \neq \emptyset .
$$

Proof: Suppose Condition (2) holds and let $B$ be a fixed set of cardinality $\mu$. Let $C^{1}, C^{2}, \ldots ; B^{\alpha}(\alpha \in \mu)$ be a partition of $B$ into $\mu$ sets $C^{p}, B^{\alpha}(p \in \mathbb{N}, \alpha \in \mu)$ each having $\mu$ elements. Now for each $\alpha \in \mu$ there exists a family $\mathcal{F}_{\alpha}$ of at most $\mu$ functions from $A_{\alpha}=\{\beta \in \mu: \beta \leqslant \alpha\}$ into $\mathbb{N}$ which is "dominating" in the sense of Condition (2). Let $\left\{B^{\alpha f}\right\}_{f \in \mathcal{F}_{\alpha}}$ be a partition of $B^{\alpha}$ into $(\leqslant \mu)$ sets each having $\mu$ elements; let

$$
B_{\alpha 1}=\left(\bigcup\left\{B^{\beta}: \beta<\alpha\right\}\right) \cup\left(\bigcup\left\{B^{\beta f}: \beta \geqslant \alpha, f \in \mathcal{F}_{\beta}, f(\alpha)=1\right\}\right) \cup C^{1}
$$

and let

$$
B_{\alpha k}=\left(\bigcup\left\{B^{\beta f}: \beta \geqslant \alpha, f \in \mathcal{F}_{\beta}, f(\alpha)=k\right\}\right) \cup C^{k} \quad(k=2,3, \ldots) .
$$

Given any $\alpha \in \mu$, one easily verifies that the sets $B_{\alpha 1}, B_{\alpha 2}, \ldots$ are pairwise disjoint, each has $\mu$ elements and their union contains each $C^{p}(p \in \mathbb{N})$ and each $B^{\beta}(\beta \in \mu)$, so that their union is $B$. Finally, let $g: \mu \rightarrow \mathbb{N}$ and $\alpha_{0} \in \mu$ be fixed and suppose we have $\alpha, k, \beta$ and $f$ such that

$$
\alpha \leqslant \alpha_{0}, 1 \leqslant k \leqslant g(\alpha), \beta \geqslant \alpha \text { and } f \in \mathcal{F}_{\beta} \text { with } f(\alpha)=k .
$$

Choose $f_{0} \in \mathcal{F}_{\alpha_{0}}$ such that $f_{0}(\delta)>g(\delta)$ for $\delta \leqslant \alpha_{0}$. Then if $\beta \neq \alpha_{0}$ we have $B^{\beta f} \subset B^{\beta}$, which is disjoint from $B^{\alpha_{0}} \supset B^{a_{0} f_{0}}$, and if $\beta=\alpha_{0}$ then $B^{\beta f}=B^{\alpha_{0} f}$, which is disjoint from $B^{\alpha_{0} f_{0}}$, since $f_{0} \neq f$. (Note that $f(\alpha)=k \leqslant g(\alpha)<f_{0}(\alpha)$ ) Thus in any case $B^{\beta f}$ is disjoint from $B^{\alpha_{0} f_{0}}$ as then is $B_{a k}$, since $\beta$ and $f$ were arbitrary, subject only to the conditions $\beta \geqslant \alpha, f(a)=k$. But $\alpha\left(\leqslant \alpha_{0}\right)$ and $k(\leqslant g(\alpha))$ arbitrary now implies that

$$
B \backslash \bigcup\left\{B_{a k}: \alpha \leqslant \alpha_{0}, k \leqslant g(\alpha)\right\} \supset B^{\alpha_{0} f_{0}} \neq \emptyset .
$$


Conversely, suppose $B_{a p}(\alpha \in \mu, p \in \mathbb{N})$ are given and let $A$ be a set of cardinality less than $\mu$. Condition (2) holds trivially for $A=\emptyset$. If $A$ is nonempty we may assume that for some $\alpha \in \mu, A=\{\beta: \beta \leqslant \alpha\}$. Then for each $g: A \rightarrow \mathbb{N}$ we can choose $x_{g} \in B \backslash \bigcup\left\{B_{\beta k}: \beta \leqslant \alpha, k \leqslant g(\beta)\right\}$ and define $f_{x_{g}}: A \rightarrow \mathbb{N}$ by letting $f_{x_{g}}(\beta)$ be the unique $p \in \mathbb{N}$ such that $x_{g} \in B_{\beta p}(\beta \leqslant \alpha)$. Clearly, then, $f_{x_{g}}(\beta)>g(\beta)$ for each $g$ and each $\beta \leqslant \alpha$, and if $x_{g}=x_{h}$ for $g$ and $h$ possibly different, we still have $f_{x_{g}}=f_{x_{h}}$, so that the cardinality of $\mathcal{F}=\left\{f_{x_{g}}: g\right.$ maps $A$ into $\left.\mathbb{N}\right\}$ cannot exceed $\mu$, the cardinality of $B$.

\section{Fit and flat components}

An application of the Set-theoretic Lemma yields our main result, for which we require considerably less than barrelledness. In fact, precisely what we require is the very weakest of all the weak barrelledness conditions currently under study by Saxon and Sánchez Ruiz, strictly weaker than Property (S) or dual locally completness, for example.

DEFINITION: A locally convex space $E$ has property $\left.f\right|_{L_{n}}$ if any given linear functional is continuous whenever its restrictions are continuous on each member of some increasing sequence of linear subspaces covering $E$.

THEOREM 1. Let $E$ be a locally convex space with property $\left.f\right|_{L_{n}}$. Suppose $B \cup C$ is a Hamel basis for $E$ with $|B|=\mu \geqslant \aleph_{0}, B \cap C=\emptyset$ ( $C$ possibly empty), where the linear coefficient functionals corresponding to elements of $B$ are all discontinuous. Then under Condition (2) for $\mu$ there is a dense $\mu$-codimensional subspace $E_{0}$ of $E$ of the form $E_{0}=s p\left(B_{0} \cup C\right)$ with $B_{0} \subset B$.

Proof: Let $B_{a p}(\alpha \in \mu, p \in \mathbb{N})$ be as in the Set-theoretic Lemma. We inductively define a function $g: \mu \rightarrow \mathbb{N}$ and choose a family $\left\{x_{\alpha}\right\}_{\alpha \in \mu} \subset B$ such that for each $\alpha \in \mu$ :

(i) $x_{\alpha} \in \bigcup_{k \leqslant g(a)} B_{a k} \backslash \bigcup\left\{B_{\beta k}: \beta<\alpha, k \leqslant g(\beta)\right\}$, and

(ii) $x_{\alpha} \in \overline{s p}\left(C \cup \bigcup_{k \leqslant g(\alpha)} B_{a k} \backslash\left\{x_{\alpha}\right\}\right)$.

Let $\alpha_{0} \in \mu$, and suppose $g(\alpha) \in \mathbb{N}$ and $x_{\alpha} \in B$ have been chosen for each $\alpha<\alpha_{0}$ so that (i) and (ii) hold. Choose $\hat{g}: \mu \rightarrow \mathbb{N}$ such that $\hat{g}(\alpha)=g(\alpha)$ for $\alpha<\alpha_{0}$. The Set-theoretic Lemma guarantees some $x_{\alpha_{0}} \in B \backslash \bigcup\left\{B_{\beta k}: \beta \leqslant \alpha_{0}, k \leqslant \widehat{g}(\beta)\right\}$. Let $f$ denote the discontinuous linear functional which is 1 at $x_{\alpha_{0}}$ and 0 elsewhere in $B \cup C$. Set

$$
E_{p}=\operatorname{sp}\left(C \cup \bigcup_{k \leqslant p} B_{a_{0} k}\right)
$$


so that $\left\{E_{p}\right\}_{p=1}^{\infty}$ is an increasing sequence of subspaces covering $E$. The hypothesis on $E$ ensures that $\left.f\right|_{E_{p}}$ is discontinuous for some $p$. Choose $q \geqslant p$ so that $x_{a_{0}} \in E_{q}$ and define $g\left(\alpha_{0}\right)=q$. Now (i) and (ii) clearly hold for $\alpha=\alpha_{0}$, completing the transfinite induction, so that $g: \mu \rightarrow \mathbb{N}$ and $\left\{x_{\alpha}\right\}_{\alpha \in \mu} \subset B$ exist with (i) and (ii) holding for all $\alpha \in \mu$.

$$
\text { If } \alpha_{1}<\alpha_{2} \text {, then } x_{\alpha_{1}} \in \bigcup_{k \leqslant g\left(\alpha_{1}\right)} B_{\alpha_{1} k} \subset \bigcup\left\{B_{\beta k}: \beta<\alpha_{2}, k \leqslant g(\beta)\right\} \text {, and } x_{\alpha_{2}} \text { is }
$$

excluded from the latter set by (i), so $x_{\alpha_{1}} \neq x_{\alpha_{2}}$; that is, the $x_{\alpha}$ 's $(\alpha \in \mu)$ are distinct. This also shows that

$$
C \cup B \backslash\left\{x_{\alpha}\right\}_{\alpha \in \mu} \supset C \cup \bigcup_{k \leqslant g\left(\alpha_{1}\right)} B_{\alpha_{1} k} \backslash\left\{x_{\alpha}\right\}_{\alpha \leqslant \alpha_{1}}=X ;
$$

thus if we suppose that $F=\overline{s p}\left(C \cup B \backslash\left\{x_{\alpha}\right\}_{\alpha \in \mu}\right)$ contains $x_{\alpha}$ for all $\alpha<\alpha_{1}$, we find that

$$
F \supset \overline{s p}\left(X \cup\left\{x_{\alpha}\right\}_{\alpha<\alpha_{1}}\right),
$$

which contains $x_{\alpha_{1}}$ by (ii). Since $\mu$ is well-ordered, we have $x_{\alpha} \in F$ for all $\alpha \in \mu$, so that $F=s p(C \cup B)=E$, and $E_{0}=s p\left(C \cup B \backslash\left\{x_{\alpha}\right\}_{\alpha \in \mu}\right)$ is a dense $\mu$-codimensional subspace of the desired form.

Now let $\left\{x_{i}: i \in B\right\}$ be a Hamel basis in a barrelled space $E$ and let $E=E_{C} \oplus E_{D}$ be the corresponding decomposition given by the Splitting Theorem. Certainly $E_{C}$, having its strongest locally convex topology, is flat. The restriction to $E_{D}$ of the coefficient functional corresponding to any member of $D$ is still discontinuous, and $D$ is either empty or infinite. In the first case $E_{D}=\{0\}$ is, trivially, fit. And for $D$ infinite, applying Theorem 1 with $B=D, C=\emptyset$ ensures $E_{D}$ is fit. Thus in any case we have

Theorem 2. [Assume Condition (2) generally.] Every barrelled space $E$ splits with respect to a given basis into a fit component $E_{D}$ and a flat component $E_{C}$.

Corollary 1. Under the above assumptions the codensity character of $E$ is $\operatorname{dim}\left(E_{D}\right)$ and is firm, that is, is attained.

Proof: Let $G$ be a dense subspace of $E_{D}$ with codimension in $E_{D}$ equal to $\operatorname{dim}\left(E_{D}\right)$. Then $E_{C}+G$ is a dense subspace of $E$ with codimension equal to $\operatorname{dim}\left(E_{D}\right)$.

Now if $F$ is any dense subspace of $E$, its image under the projection of $E$ onto $E_{C}$ along $E_{D}$ is dense in $E_{C}$ and thus is all of the flat $E_{C}$. Therefore $F+E_{D}=E$, and $\operatorname{codim}_{E}(F) \leqslant \operatorname{dim}\left(E_{D}\right)$.

Theorem 2 also shows that barrelled spaces that are neither fit nor flat can be constructed only as in [5]; indeed, since a non-flat barrelled space must have dimension at least $K_{1}$ we have 
Corollary 2. A barrelled space $E$ is neither fit nor flat if and only if $E=$ $E_{1} \oplus E_{2}$ (topologically), where $E_{1}$ is flat, $E_{2}$ is fit and $\operatorname{dim}\left(E_{1}\right)>\operatorname{dim}\left(E_{2}\right) \geqslant \aleph_{1}$.

The next result says the codensity character of a non-flat barrelled space is at least $\aleph_{1}$, which extends Corollary 1 of Theorem 3 in [3] and was announced without proof in [6]. This extension is optimal under our assumption that $\aleph_{1}=0$, since the metrisable (and thus fit) barrelled space $\psi_{b}$ of [7] must then have codensity character $\aleph_{1}$.

Theorem 3. [Assume $\mathfrak{d}=\aleph_{1}$.] Every non-flat barrelled space $E$ has a dense subspace of codimension at least $\aleph_{1}$.

Proof: Take $B$ any subset of a given basis for $E$ with $B \subset E_{D}$ and $|B|=\aleph_{1}$; apply Theorem 1 .

Corollary 1. [Assume $\mathfrak{d}=\aleph_{1}$.] If $E$ is a non-flat barrelled space, then

$$
\operatorname{codim}_{E^{*}}\left(E^{\prime}\right) \geqslant 2^{\aleph_{1}}
$$

Proof: By Theorem $3 E$ has an $\aleph_{1}$-codimensional dense subspace. The result then follows immediately from Theorem 4 of [3].

REMARK. Under the stronger assumption that the Continuum Hypothesis ( $\mathrm{CH}$ ) holds we get analogously that the codimension of $E^{\prime}$ in $E^{*}$ must be at least $2^{\text {c }}$; consequently, there is no barrelled space whose dual has codimension $c$ in the algebraic dual.

Now we recall two definitions from [5]. Let $\left\{x_{i}, f_{i}\right\}_{i \in B}$ be a biorthogonal system in $E \times E^{*}$ whose first coordinates form a Hamel basis in $E$. Then $\left\{x_{i}, f_{i}\right\}_{i \in B}$ is called a discontinuous basis if each $f_{i}$ is discontinuous, and a fully discontinuous basis if $\operatorname{sp}\left(\left\{f_{i}: i \in B\right\}\right) \cap E^{\prime}=\{0\}$. It is shown in Theorem 6 of [5], without any nonZFC assumptions, that any fit topological vector space has a fully discontinuous basis. Combining this with Theorem 1 we have immediately:

THEOREM 4. When $E$ has property $\left.f\right|_{L_{n}}$ the following are equivalent:

(a) $E$ is fit;

(b) E has a fully discontinuous basis;

(c) $E$ has a discontinuous basis.

Note. Generally, (a) $\Rightarrow$ (b) $\Rightarrow$ (c) and neither arrow is reversible [5].

For a barrelled space $E$ with a given basis, the fit and flat components $E_{D}$ and $E_{C}$ are uniquely defined. By Corollary 1 to Theorem 2 the dimension of $E_{D}$ is always the codensity character of $E$, which is independent of the basis. However, the topological nature of $E_{D}$ may vary widely with the choice of basis, as may the algebraic nature of $E_{C}$. To see this, let $E=M \oplus N$ be the topological direct sum of two barrelled spaces $M$ and $N$ of the same infinite dimension, with $M$ metrisable and $N$ flat, as considered 
in [5]. Let $N_{1}$ and $N_{2}$ represent arbitrary algebraic complements in $N$; they must be topologically complementary as well, since $N$ has its strongest locally convex topology, and $E$ is the topological direct sum of $M+N_{1}$ and $N_{2}$. Since $M$ is fit (indeed, $M$ is Baire-like and thus quasi-Baire) and $\operatorname{dim}(M) \geqslant \operatorname{dim}\left(N_{1}\right)$, we have $M+N_{1}$ is also fit. By Theorem 4 (or by [5, Theorem 6]) there is a (fully) discontinuous basis $B_{1}$ for $M+N_{1}$. If $B_{2}$ is any basis for $N_{2}$, then $B=B_{1} \cup B_{2}$ is a basis for $E$ with respect to which $E_{D}$ is $M+N_{1}=M \oplus N_{1}$ and $E_{C}$ is $N_{2}$. Now the choice of dimensions for $N_{1}$ and $N_{2}$ ranges maximally from 0 to $\operatorname{dim}(E)$, thus also for $E_{C}$, and $E_{D}$ is metrisable if and only if $\operatorname{dim}\left(N_{1}\right)$ is finite. In fact, all distinct infinite values for $\operatorname{dim}\left(N_{1}\right)$ yield non-isomorphic values for $E_{D}$, for if a subspace $N^{(1)}$ of $M \oplus N_{1}$ has infinite dimension exceeding $\operatorname{dim}\left(N_{1}\right)$ then $N^{(1)}$ contains an infinite-dimensional (metrisable) subspace of $M$ and cannot be flat.

\section{An application to the BCE problem}

The following problem has been extensively studied in recent years (for a survey see $[\mathbf{1 0}])$ :

Problem. Let $E$ be a non-flat barrelled space. Does there exist a subspace $M$ of $E^{*}$ such that $\operatorname{dim}(M)=\aleph_{0}, E^{\prime} \cap M=\{0\}$ and $E$ is barrelled under the Mackey topology $\tau\left(E, E^{\prime}+M\right)$ ?

When the answer is "yes" we say $E$ has a barrelled countable enlargement (BCE).

According to Eberhardt and Roelcke [2] a $G M$-space is a locally convex space $E$ such that any linear mapping $t: E \rightarrow F$, where $F$ is any metrisable locally convex space and $t$ has closed graph, is continuous. Since $F$ may be any Banach space it is immediate that GM-spaces are barrelled.

Let $E$ be a non-flat GM-space. If we assume $\mathfrak{d}=\aleph_{1}$ then Theorem 3 guarantees that $E$ has a dense $\aleph_{1}$-codimensional subspace. Since every dense subspace of a GMspace is barrelled $[2,1.9]$, Theorem 5 of $[8]$ now tells us that $E$ has a BCE. (The cardinal $b$ which appears in Theorem 5 of $[8]$ satisfies $\aleph_{1} \leqslant \mathfrak{b} \leqslant \mathfrak{d}[1,3.1]$, so that under $\mathfrak{d}=\aleph_{1}$ all three coincide.)

THEOREM 5. [Assume $\mathfrak{d}=\aleph_{1}$ ] Every non-flat $G M$-space has a $B C E$.

REMARK. In Section 4 of [4], Theorem 5 is proved, without non-ZFC assumptions, for $\aleph_{0}$-products, a subclass of the class of GM-spaces [2, Section 3]. Since $\aleph_{0}$-products are complete, their proper dense subspaces are non-flat GM-spaces which are not $\aleph_{0}$ products.

\section{REFERENCES}

[1] E.K van Douwen, 'The integers and topology', in Handbook of Set-theoretic Topology, (K. 
Kunen and J.E. Vaughan, Editors) (North-Holland, 1984), pp. 111-168.

[2] V. Eberhardt and W. Roelcke, 'Über einen Graphensatz für lineare Abbildungen mit metrisierbarem Zielraum', Manuscripta Math. 13 (1974), 53-68.

[3] W.J. Robertson, S.A. Saxon and A.P. Robertson, 'Barrelled spaces and dense vector subspaces', Bull. Austral. Math. Soc. 37 (1988), 383-388.

[4] W.J. Robertson, I. Tweddle and F.E. Yeomans, 'On the stability of barrelled topologies III', Bull. Austral. Math. Soc. 22 (1980), 99-112.

[5] S.A. Saxon, 'The codensity character of topological vector spaces', in Topological vector spaces, algebras and related areas, (A. Lau and I. Tweddle, Editors) (Longman, 1994), pp. 24-36.

[6] S.A. Saxon and W.J. Robertson, 'Dense barrelled subspaces of uncountable codimension', Proc. Amer. Math. Soc. 107 (1989), 1021-1029.

[7] S.A. Saxon and L.M. Sánchez Ruiz, 'Optimal cardinals for metrizable barrelled spaces', J. London Math. Soc. (to appear).

[8] S.A. Saxon and L.M. Sánchez Ruiz, 'Barrelled countable enlargements and the bounding cardinal', J. London Math Soc. (to appear).

[9] S.A. Saxon and L.M. Sánchez Ruiz, 'Barrelled countable enlargements and the dominating cardinal', (preprint).

[10] I. Tweddle, S.A. Saxon and L.M. Sánchez Ruiz, 'Barrelled countable enlargements', in Topological vector spaces, algebras and related areas, (A. Lau and I. Tweddle, Editors) (Longman, 1994), pp. 3-15.

Department of Mathematics

University of Florida

PO Box 118000

Gainesville FL 32611-8000

United States of America

\author{
Department of Mathematics \\ University of Strathclyde \\ Glasgow G1 1XH \\ Scotland \\ United Kingdom
}

